\title{
L'action des syndicats agricoles en faveurde l'installation dans les Commissions départementales d'orientation agricole
}

The action of the agricultural trade unions for the setting-up in the Departmental Commissions of Agricultural Orientation

Valérie Bernardi et Jean-Pierre Boinon

\section{(2) OpenEdition}

\section{Journals}

Édition électronique

URL : http://journals.openedition.org/economierurale/856

DOI : 10.4000/economierurale.856

ISSN : 2105-2581

\section{Éditeur}

Société Française d'Économie Rurale (SFER)

Édition imprimée

Date de publication : 10 août 2009

Pagination : 80-92

ISSN : 0013-0559

Référence électronique

Valérie Bernardi et Jean-Pierre Boinon, "L'action des syndicats agricoles en faveurde l'installation dans les Commissions départementales d'orientation agricole », Économie rurale [En ligne], 312 I Juillet-août 2009, mis en ligne le 05 juillet 2011, consulté le 01 mai 2019. URL : http:// journals.openedition.org/economierurale/856 ; DOI : 10.4000/economierurale.856 


\section{L'action des syndicats agricoles en faveur de l'installation dans les Commissions départementales d'orientation agricole}

Valérie BERNARDI - Centre national pour l'aménagement des structures des exploitations agricoles (CNASEA), Direction de la communication et des études (DCE)

Jean-Pierre BOINON • Centre d'Économie et de Sociologie Appliquée à l'Agriculture et aux Espaces Ruraux (CESAER) Agro Sup Dijon

D epuis les années 1960, les syndicats agricoles majoritaires (Fédération nationale des syndicats d'exploitants agricoles - FNSEA - et Centre national des jeunes agriculteurs - CNJA) sont étroitement associés à la définition et à la mise en œuvre des politiques agricoles, cette cogestion de l'agriculture ayant été élargie aux syndicats minoritaires (Confédération paysanne, Coordination rurale et Mouvement de défense des exploitants familiaux - Modef) au début des années 80 avec l'arrivée de la gauche au gouvernement (Coulomb, 1990). Au niveau départemental, plusieurs commissions alliant représentants syndicaux et représentants de l'administration de l'agriculture ont été mises en place pour définir les orientations départementales des politiques agricoles et les mettre en œuvre au quotidien. En 1995, les Commissions départementales d'orientation de l'agriculture (CDOA) se sont vues confier des pouvoirs très étendus, allant de la politique d'installation et de modernisation jusqu'au contrôle des structures et à la gestion des réserves départementales de droits à produire et à prime (Barthélemy et David, 1999). Parce qu'elles ont la possibilité d'orienter l'évolution des structures des exploitations agricoles d'un département, les CDOA représentent un enjeu fort pour le syndicalisme agricole et l'attribution des sièges représentant les différentes organisations professionnelles agricoles dépend du résultat des élections aux Chambres d'agriculture.
Cet article s'appuie sur une étude concernant la place et le poids des CDOA dans les politiques départementales d'installation des jeunes agriculteurs, menée par le Centre national pour l'aménagement des structures des exploitations agricoles (CNASEA) en 2004 et 2005, c'est-à-dire pendant la période de préparation de la loi d'orientation agricole du 5 janvier 2006, qui a fortement atténué les règles en vigueur jusqu'alors pour le contrôle des structures. Dans la première partie, nous présenterons la construction dans un cadre national de la politique d'installation des jeunes agriculteurs en soulignant le rôle déterminant du CNJA dans la définition de cette politique. À partir d'une étude de la mise en œuvre des politiques d'installation par les CDOA dans six départements (Machline, 2005), nous analyserons dans la deuxième partie la manière dont chaque CDOA mobilise les moyens réglementaires à sa disposition pour améliorer le taux d'installation. Enfin, dans la troisième partie le rôle des différents syndicats agricoles dans l'élaboration et la mise en œuvre des politiques départementales d'installation sera examiné.

\section{Une politique d'installation inscrite dans la politique des structures}

L'accès au foncier est souvent considéré comme l'obstacle principal à franchir pour celui qui veut s'installer en agriculture, et son principal concurrent est l'agriculteur déjà installé qui souhaite s'agrandir. Dès la Première Guerre mondiale, le Syndicat 
national des migrations rurales est préoccupé par le fait qu'il existe dans certaines régions « des paysans sans terres » et dans d'autres «des terres sans paysans ». Ainsi s'organisent les premières migrations d'agriculteurs, principalement en provenance de l'Ouest vers la Bourgogne, le Limousin et le Sud-Ouest.

La France du début des années 1960 se donne comme objectifs le développement de l'autosuffisance alimentaire, la réduction du déficit commercial agricole et l'amélioration des conditions de vie des paysans. Il faut donc un système d'exploitation efficiente et à forte productivité. Dans un contexte de fort exode rural, les lois d'orientation agricole de 1960 et 1962 définissent un modèle d'exploitation de type familial : «L'exploitation à deux unités de travail humain (UTH) », dans le but, d'une part, d'encourager le départ d'agriculteurs disposant de trop petites structures de production et, d'autre part, d'orienter les surfaces libérées vers les agriculteurs qui correspondent au modèle visé. La politique des structures mise en place à cette époque est essentiellement marquée par des actions concernant la cessation d'activité des agriculteurs : indemnité viagère de départ, aides en faveur des mutations professionnelles.

Dès le début des années 1970, la politique de modernisation de l'agriculture et d'encouragement à l'exode agricole a très rapidement connu des limites, notamment dans les zones difficiles où le maintien d'un minimum de peuplement et d'une activité agricole suffisante pour entretenir l'espace naturel n'était pas assuré. La politique d'aide à l'installation des jeunes agriculteurs est élaborée au début des années 70 sous l'impulsion du CNJA, dans la continuité de la politique des structures mise en place par les lois d'orientation agricole du début des années 60, car vouloir favoriser l'installation nécessite dès lors un contrôle rigoureux des agrandissements. C'est dans ce contexte qu'en
1973 est instaurée en France une aide spécifique, la «Dotation jeune agriculteur » (DJA), destinée aux jeunes âgés de moins de 35 ans s'engageant à être agriculteurs pendant cinq ans dans les zones de montagne et défavorisées. Cette aide est étendue trois ans plus tard à l'ensemble du territoire national, mais son montant peut être modulé selon le lieu de l'exploitation d'installation (montagne, zone défavorisée ou plaine) et selon des critères qui tiennent compte d'éléments plus ou moins favorables dans la situation du jeune qui s'installe. L'objectif affiché de cette aide est alors de faciliter la transmission des exploitations et de donner au jeune agriculteur l'autonomie financière qui lui permettra de racheter le patrimoine, souvent familial, nécessaire à la constitution de son entreprise agricole et ainsi affirmer l'autonomie de l'entreprise créée par rapport au patrimoine familial (Barthélemy, 2001). La politique d'installation des jeunes agriculteurs qui n'a cessé d'être soutenue par le CNJA, a progressivement gagné en importance et a été reprise en tant qu'objectif majeur de politique agricole par les principales organisations syndicales agricoles (FNSEA, Confédération paysanne).

Depuis cette période, les critères pour obtenir les aides à l'installation ont été renforcés en vue de promouvoir une exploitation agricole familiale compétitive et rémunérant de manière satisfaisante les travailleurs familiaux. Dans les années 80 , l'accent est mis sur la nécessité d'une approche économique. Sous l'impulsion du CNJA, un critère de revenu remplace celui de surface et un décret de 1988 introduit l'obligation de réaliser une Étude prévisionnelle d'installation (EPI). Cette EPI doit faire apparaître que l'installation prévue permettra au jeune d'obtenir dans les trois ans qui suivent son installation un revenu comparable à celui qu'il aurait obtenu dans un autre secteur d'activité. La capacité professionnelle demandée est augmentée, tandis que le public est 
étendu aux conjoints (l'activité professionnelle des conjoints d'exploitant est ainsi reconnue) et aux pluriactifs, dans le but de contribuer au maintien d'un tissu rural dans les secteurs difficiles. Les caractéristiques principales du dispositif ont peu évolué depuis. Ainsi, les obligations de capacité professionnelle et de justification d'un revenu qui puissent être comparé à celui obtenu dans d'autres secteurs d'activité témoignent d'une démarche syndicale visant à la reconnaissance professionnelle du métier d'agriculteur et à la nécessaire rémunération du travail de l'agriculteur. Les renforcements successifs de ces critères excluent du bénéfice des aides un nombre croissant d'agriculteurs qui s'installent en dehors des normes exigées. On a pu évaluer à $1 / 3$ les installations qui se réalisaient sans bénéficier d'aides au début des années 90 (Rémy, 1997). En 2000, les statistiques comparées de la Mutualité sociale agricole (MSA) et du CNASEA montraient que $36 \%$ des nouveaux exploitants de moins de 40 ans ne bénéficiaient pas des aides à l'installation.

Si les objectifs assignés à la politique d'installation des jeunes agriculteurs sont clairement définis et en cohérence avec les autres politiques agissant sur les structures des exploitations agricoles, les règles et modalités d'application sont définies à un niveau départemental. L'application de ces politiques à un niveau local s'est inscrite dans une logique, définie au cours des années 70, de cogestion de la politique agricole entre les pouvoirs publics et les organisations professionnelles jugées représentatives (Coulomb, 1990, Vercherand, 2009). Les formes administratives de cette cogestion sont assez complexes alliant les représentants de l'État, essentiellement les Directions départementales de l'agriculture et de forêt (DDAF), aux organisations professionnelles agricoles (Chambre d'agriculture, syndicalisme agricole majoritaire, instituts de développe- ment technique, organisations coopératives et de crédit...). En matière d'installation et d'orientation des structures des exploitations agricoles, trois commissions ont alors une importance particulière :

- Le Comité technique départemental des Sociétés d'aménagement foncier et d'établissement rural (SAFER), chargé d'appliquer la politique de rétrocession des terres acquises par les SAFER. Jusqu'en 1999, ce comité technique était composé par les actionnaires des SAFER, c'est-àdire des syndicats agricoles majoritaires et des organisations professionnelles qui leur étaient liées, auxquels s'ajoutaient deux commissaires du gouvernement chargés de veiller à la légalité des décisions de ce comité technique. Depuis 1999, les représentants des syndicats agricoles minoritaires peuvent siéger dans ce comité.

- La Commission des structures, chargée de la mise en œuvre de la politique des structures, composée de représentants de l'État et de la profession agricole.

- La Commission mixte, également composée de représentants de l'État et de la profession agricole, chargée de la mise en œuvre de la politique d'installation et de modernisation, et à partir de la fin des années 80, chargée de la gestion des réserves départementales de quotas laitiers et de droits à primes.

Ces deux dernières commissions ont été fusionnées en 1995, pour donner naissance à la CDOA aux compétences élargies à la définition des orientations de l'agriculture départementales. L'objectif de la réunion, dans une même commission des compétences de la commission des structures et de la commission mixte, était de renforcer la cohérence des décisions en matière d'orientation des structures des exploitations agricoles du département (Berriet-Solliec et Boinon, 2000). 


\section{Le rôle des Commissions départementales dans les politiques d'installation}

Placée sous la présidence du Préfet, les CDOA comprennent des représentants de l'administration, des collectivités locales, des organisations professionnelles agricoles, du secteur de l'économie agricole et, depuis 1999, des associations de consommateurs et de protection de l'environnement. Ces commissions ont pour mission de définir les orientations départementales en matière d'évolution des structures de production agricole, et notamment l'orientation du foncier libéré par les départs d'agriculteurs vers l'installation de jeunes agriculteurs ou vers l'agrandissement des exploitations agricoles existantes. Cette orientation de l'agriculture est réalisée principalement à travers le contrôle des structures, la gestion des réserves départementales de droit à produire, l'application des politiques de modernisation et d'installation. C'est ce que nous allons voir dans le premier point après avoir exposé ce que représente le «taux de renouvellement » en agriculture. Un éclairage particulier sur l'action de ces commissions dans six départements français fera l'objet du second point.

\section{Les divers outils en faveur de l'installation}

L'évolution de la population agricole se caractérise par un nombre important de départs à la retraite et par une baisse des naissances. Or, pour l'essentiel, ce sont les fils et filles d'agriculteurs qui s'installent et ceux-ci ne suffisent pas à combler les départs en masse. Le faible taux de renouvellement des exploitants agricoles (rapport entre le nombre des premières immatriculations comme chef d'exploitation à la MSA et le nombre de sorties de l'agriculture recensées par la MSA) fragilise les territoires les plus sensibles. Si ce taux a nettement progressé de 1993 à 1998, passant de $25 \%$ à plus de $50 \%$, il s'est en revanche stabilisé sur la période récente entre 50 et $60 \%$ (54\% en 2002) (Cahiers du CNASEA, 2002). Cette stabilisation est liée, à la diminution du nombre de sorties (Lefevre, 2004).

Mais ce taux de renouvellement des chefs d'exploitation présente de fortes disparités régionales. Trois groupes de régions atteignent des taux élevés :

- à l'ouest la Bretagne, à l'exception du Finistère et des Pays de la Loire ;

- au sud un groupe de six départements voisins (Aveyron, Cantal, Gard, HauteLoire, Lot et Lozère) ;

- au nord et à l'est : une large bande partant de la Haute-Normandie et de la Picardie pour atteindre le nord de la Bourgogne et de la Franche-Comté, en passant par la Champagne-Ardenne et la Lorraine.

A contrario, les régions PACA, Corse et Rhône-Alpes d'une part, et une bande s'étalant de l'Ile-de-France aux Landes via la région Centre d'autre part, ont des taux de renouvellement inférieurs à $50 \%$ voire $40 \%$ (Cahiers du CNASEA, 2002).

Le taux d'installation aidée est en moyenne de $64 \%$ sur la période 1997-1999. Il présente, lui aussi, de fortes disparités territoriales. Enfin, les taux d'installation hors du cadre familial les plus élevés sont situés dans les départements du pourtour méditerranéen, de Rhône-Alpes, FrancheComté et de l'ouest, tandis que le quart nord-est de la France présente les taux les plus faibles.

Les CDOA disposent de différents moyens opérationnels pour mener à bien leur politique d'installation et assurer ainsi un taux de renouvellement suffisant des chefs d'exploitation sur le territoire français. Les outils en lien direct avec l'installation sont :

- le contrôle des structures ;

- la gestion des réserves départementales de droits à prime et à produire ;

- la modulation de la DJA. 
Le contrôle des structures

C'est l'outil principal à la disposition des CDOA pour éviter la concentration des terres. La première version de ce qui s'appelait alors le « contrôle des cumuls » a été votée en 1962 et a été popularisée par la manifestation de 700 agriculteurs de l'Orne devant le domicile normand de l'acteur Jean Gabin, qui possédait quatre exploitations agricoles travaillées par des salariés, alors que les jeunes agriculteurs normands trouvaient difficilement des terres à exploiter (Debatisse, 1963). Cette réglementation concrétise les notions de surface minimum, ou seuil de viabilité en dessous duquel l'installation n'est pas encouragée et de surface maximum au-delà de laquelle une autorisation d'exploiter est nécessaire. Le contrôle des cumuls deviendra « contrôle des structures » avec la loi du 4 juillet 1980 qui fixe pour chaque département l'obligation d'élaborer un Schéma directeur départemental des structures $(\mathrm{SDDS})^{1}$. Les axes prioritaires du contrôle des structures sont fixés par le SDDS, qui fait l'objet d'un arrêté préfectoral et établit les normes du contrôle dans le département. Il précise en particulier :

- l'unité de référence (UR) fixée par référence à la moyenne des installations aidées sur les cinq dernières années, qui remplace la Surface minimale d'installation (SMI), et tient compte des équivalences pour les élevages hors sol et pour les cultures spéciales ;

- le seuil de déclenchement qui pouvait être décidé au moment de notre étude entre 0,5 et 1,5 fois $1^{\prime} \mathrm{UR}^{2}$;

- le seuil de démembrement qui peut être déterminé entre $1 / 3$ et 1 fois l'UR ;

- la distance d'éloignement des terres par rapport au centre de l'exploitation, qui doit être supérieure à cinq kilomètres.

1. Article L.312-1 du Code rural.

2. La loi du 5 janvier 2006 a relevé le seuil «entre une et deux fois l'unité de référence ».
Le contrôle des structures définit les cas pour lesquels l'obtention d'une autorisation d'exploiter est nécessaire. Sont en particulier soumises à autorisation préalable : - les installations, agrandissements ou réunion d'exploitations dont la surface dépasse le seuil de déclenchement ;

- la diminution du nombre d'associés si l'exploitation présente une surface supérieure au seuil de déclenchement ;

- les agrandissements ou réunion d'exploitations si la distance au siège est supérieure à la distance d'éloignement fixée par le SDDS ;

- les installations ou agrandissements ayant pour effet de supprimer, ou de ramener une exploitation dont la surface est supérieure au seuil de démembrement en dessous de ce seuil.

Le SDDS fixe surtout un ordre de priorité à l'attribution des autorisations d'exploiter, qui s'impose aux organes du contrôle que sont le Préfet et la CDOA. Certes la loi exige que la première des priorités soit l'installation devant l'agrandissement, cependant il existe une marge de manœuvre dans la subdivision des priorités et permet l'introduction d'autres critères de décision. L'acceptation, ou le refus de délivrance de l'autorisation, doit être justifié au regard de cet ordre de priorité en fonction des concurrences en présence.

La dotation des droits à prime et à produire Le régime des quotas laitiers a été instauré en 1984 afin de limiter les excédents de produits laitiers au sein de la Communauté européenne. Le quota peut se définir comme la quantité de lait qu'un producteur peut livrer annuellement à un acheteur sans subir de pénalité financière. Ces quantités sont réparties en références individuelles au niveau des producteurs. Contrairement aux autres pays européens, la France a choisi un mode de gestion non marchand et administré des droits à produire et à prime. L'État constitue des réserves de droits à produire 
qui sont gérées au niveau départemental par les CDOA. En France, les quotas laitiers sont liés au foncier et tout mouvement de foncier entraîne un transfert de quota. Pour en bénéficier, un jeune agriculteur doit reprendre des terres avec quotas, mais aussi obtenir des quotas supplémentaires grâce aux réserves nationale et départementale.

La réserve départementale est constituée par $80 \%$ des cessations primées, ainsi que l'intégralité des cessations spontanées, des sous réalisations structurelles (depuis la campagne 2000-2001) et des prélèvements lors des transferts fonciers. La réserve nationale correspond à une mutualisation en faveur des petits producteurs. Elle est alimentée par $20 \%$ des cessations primées, et distribuée aux départements selon le nombre de jeunes agriculteurs installés au cours des années précédentes ainsi que selon le nombre de petits producteurs ayant une référence inférieure à 100000 litres. Sa redistribution au niveau départemental vise ces deux catégories et est assurée par la CDOA.

La marge de manœuvre de la CDOA en matière de gestion des quotas laitiers dépend de l'importance de la réserve constituée. La CDOA dispose d'une grande liberté pour la gestion et la redistribution des quotas des réserves départementale et nationale entre les repreneurs de foncier, les jeunes agriculteurs qui s'installent ou l'attribution à d'autres producteurs. La CDOA décide ensuite de la façon dont est répartie la réserve départementale entre les attributaires potentiels. La CDOA peut donc choisir d'orienter de façon plus ou moins forte sa politique en direction de l'installation laitière, en modulant les montants de dotation à l'installation à partir de la réserve constituée.

En ce qui concerne les droits à prime dans les secteurs des viandes bovines et ovines, depuis le $1^{\text {er }}$ juillet 1992 , les producteurs bovins et ovins doivent détenir des droits pour accéder à la distribution des primes communautaires, que sont la prime au maintien des troupeaux de vaches allai- tantes (PMTVA) et la prime à la brebis et à la chèvre $(\mathrm{PBC}$, anciennement $\mathrm{PCO}$, prime compensatoire ovine). De la même façon que pour les quotas laitiers, la marge de manœuvre de la CDOA dépend de l'importance de la réserve constituée, qui est alimentée principalement à l'occasion des démembrements d'exploitation dans lesquels les droits à prime sont en totalité prélevés par la réserve. Dans le cas d'une transmission totale de l'exploitation (procédure de cession-reprise), aucun droit n'est prélevé pour la réserve départementale. Tout comme pour les quotas laitiers, l'attribution de droits supplémentaires à un éleveur est décidée par la CDOA en fonction de la politique qu'elle a définie.

\section{La modulation de la dotation jeune agriculteur}

La modulation de la dotation jeune agriculteur (DJA) est une aide à l'installation financée par l'État et l'Union européenne dans le cadre du Règlement de développement rural, qui vise à compléter la trésorerie nécessaire au démarrage de l'activité agricole. Toute personne désireuse de s'installer peut être attributaire de la DJA si elle entre dans les conditions générales d'attribution. Ces conditions sont relatives à la personne, à la préparation du projet d'installation ainsi qu'à d'autres engagements (Hottlet et CNJA, 2001). Chaque dossier de DJA est examiné en CDOA. Son montant est modulé sur la base de critères fixés par la CDOA dans les limites comprises entre $8000 €$ (DJA minimum en zone de plaine) et $35900 €$ (DJA maximum pour les zones de montagne). La CDOA peut définir, au sein d'une zone, les critères pouvant majorer ou minorer le montant de l'aide.

La CDOA a donc à la fois un pouvoir de contrôle et un pouvoir d'orientation : le premier concerne la dimension foncière de l'exploitation, via le contrôle des structures, qu'elle peut décider de plus ou moins renforcer selon les seuils qu'elle fixe. Le second concerne la dimension économique 
de l'exploitation : d'une part, la CDOA peut choisir de majorer l'aide à l'installation pour certains types d'exploitation selon des critères qu'elle a préalablement fixés (par exemple pour les installations hors du cadre familial) et, d'autre part, il existe pour chacun des droits à produire ou à prime dont elle a la gestion, une marge de manœuvre dans la proportion de la réserve qu'elle choisit d'orienter vers l'installation.

\section{L'utilisation des outils réglementaires Un éclairage sur six départements}

Alors que les commissions départementales affichent toutes la priorité de l'installation dans leur Projet agricole départemental (PAD) dans quelles mesures mobilisentelles les moyens réglementaires à leur disposition pour améliorer le taux d'installation? C'est à cette question que nous avons cherché à répondre en effectuant un éclairage sur six départements. Le choix de ces départements s'est effectué de telle façon qu'il nous permette d'effectuer des comparaisons « deux à deux ». Pour cela nous avons choisi deux entrées principales :

- la situation géographique du département et le type de production ;

- le taux d'installation dans le département.

Pour la première entrée, nous avons ainsi choisi deux départements à orientation grandes cultures, deux départements à dominante laitière, et deux départements orientés vers l'élevage allaitant. Ont été délibérément exclues certaines situations qui risquaient d'induire des biais importants dans l'étude, tels que les départements où la vigne est très présente (les droits de plantation ne sont pas gérés par la CDOA), les départements de haute montagne qui sont soumis à des contraintes très spécifiques ou ceux où la pression hors-agricole sur le foncier domine.

En ce qui concerne la seconde entrée, pour chaque département le taux de renouvellement des chefs d'exploitation sur la période 1998-2003 a été calculé. Le seuil de
$40 \%$ pour le taux de renouvellement a été choisi pour différencier les départements entre ceux qui ont des bons résultats en matière d'installation et les autres.

Enfin on a pris en compte l'étude sur les Projets agricoles départementaux (BerrietSolliec, Boinon, 2000), en privilégiant des départements dont le PAD présente une bonne qualité de rédaction relativement à la situation départementale, aux objectifs et aux moyens.

En tenant compte de ces différents critères, notre choix s'est finalement porté sur :

- l'Aveyron et la Haute-Vienne, départements à dominante d'élevage allaitant, qui différaient par leur taux de renouvellement fort en Aveyron (49\%) et plus faible en Haute-Vienne (28\%) ;

- le Morbihan et le Finistère, départements à dominante bovin lait, qui différaient par leur taux de renouvellement fort dans le Morbihan (43\%) et plus faible dans le Finistère $(36 \%)$;

- la Somme et l'Eure, départements à dominante grandes cultures, avec un taux de renouvellement élevé dans la Somme (54\%), et plus faible dans l'Eure (39\%).

Le contrôle des structures s'impose comme l'outil central des CDOA. Les départements de l'Aveyron, de la Somme et du Morbihan, où les taux de renouvellement sont les plus élevés parmi les départements étudiés, ont mis en place une politique des structures efficace.

- Dans l'Aveyron, il apparaît clairement que tous les moyens sont mis en œuvre pour que le contrôle des structures puisse être efficace : le seuil de déclenchement est bas (0,75 UR), l'implication des syndicats au niveau local est forte, et les dossiers des candidats sont comparés grâce à un système d'évaluation du potentiel économique des exploitations, au sein de catégories de priorité détaillées. Il y a une recherche systématique de tous les candidats potentiels par le travail local des 
structures syndicales, relayées par les différents organismes professionnels.

Comparativement, la Haute-Vienne ne met pas en place les conditions qui peuvent rendre le contrôle des structures efficace. Le seuil de déclenchement du contrôle y est élevé (1,2 UR), l'implication locale est faible et l'autorisation d'exploiter est donnée à tous les candidats dès lors qu'ils entrent dans la même catégorie de priorité, catégories qui sont d'ailleurs peu détaillées. La gestion des droits PMTVA apparaîtrait plus favorable à l'installation dans la HauteVienne, puisque $75 \%$ de la réserve est attribuée aux jeunes agriculteurs, contre $40 \%$ dans l'Aveyron. Cependant, les réserves sont très insuffisantes par rapport à la demande, car la grande majorité des échanges de droits se fait en réalité par la procédure de cession-reprise, qui ne peut être contrôlé que par une application plus stricte du contrôle des structures. Aussi, la façon dont est mis en œuvre le contrôle des structures en Haute-Vienne en limite fortement l'efficacité. À l'opposé, en Aveyron, le contrôle des structures intervient davantage sur l'agrandissement des exploitations de bovins allaitants par cession-reprise.

- Pour la Somme et l'Eure, départements de grande culture, les politiques des CDOA diffèrent nettement dans leur manière d'appliquer le contrôle des structures. La Somme a un seuil bas de déclenchement du contrôle des structures (0,5 UR), et le réseau FDSEA-JA fait remonter des avis sur presque toutes les demandes d'autorisation d'exploiter (ce sont d'ailleurs la FDSEA et les JA qui souhaitent le maintien d'un seuil de déclenchement bas, contre l'avis de la Coordination rurale et du Syndicat de la propriété agricole). Dans l'Eure la Confédération paysanne dénonce le seuil de déclenchement élevé (1,2 UR), qui selon elle décourage les candidats potentiels à faire des demandes concurrentes. La volonté de limiter l'agrandissement des exploitations dans la Somme pour favoriser l'installation de jeunes agriculteurs a entraîné une orientation des systèmes de production vers une utilisation plus intensive de la terre (légumes, pommes de terre, betterave...), orientation favorisée par la forte présence de l'industrie agroalimentaire.

- Pour le Morbihan et le Finistère, à orientation laitière, sont les deux départements où le nombre de dossiers traités chaque année en contrôle des structures est le plus important, et où les taux de concurrence sont les plus élevés. Ils diffèrent par le niveau du seuil de déclenchement (0,5 UR dans le Morbihan, 1 UR dans le Finistère). Mais la gestion de la réserve départementale des quotas laitiers est très différente entre les deux départements, et ce dès la constitution de la réserve qui, dans le Finistère, est en partie réattribuée aux repreneurs de fonciers. Le discours de la FDSEA et des JA est à ce sujet paradoxal : ils affirment vouloir conforter l'installation mais sans trop prélever, considérant que la réattribution des quotas aux repreneurs n'empêche pas la constitution d'une réserve conséquente. La Confédération paysanne, quant à elle, considère que l'allègement des prélèvements encourage les grosses exploitations à la reprise de foncier, augmentant ainsi la pression foncière au détriment des candidats à l'installation. Dans le Morbihan, les prélèvements de quotas laitiers lors de transferts fonciers sont systématiques, ce qui permet une plus grande disponibilité dans la réserve départementale et ainsi une plus grande possibilité d'attribution de quotas laitiers pour les jeunes agriculteurs qui s'installent.

Dans tous les départements, l'efficacité de la gestion des droits à prime et quotas est conditionnée à celle du contrôle des structures, en raison du lien au foncier dans le cas des quotas laitiers et parce que, dans le cas des PMTVA, le contrôle des structures offre la seule possibilité d'intervention sur les agrandissements par cession reprise. Mais le refus d'une autorisation d'exploiter contre l'avis du propriétaire, dans le but d'attribuer des terres à un jeune qui s'installe, ne garantit en aucun cas la signature du bail : 
le propriétaire a le choix de ne pas louer, ou de vendre ses terres. Ces situations de blocage limitent partout l'impact du contrôle des structures. L'opposition entre les syndicats d'exploitants et la Propriété agricole est d'ailleurs plus forte là où le contrôle des structures est mis en œuvre de façon interventionniste. Ces blocages constituent néanmoins le prix à payer pour une certaine efficacité, puisque parfois l'avis des propriétaires évolue en faveur de l'installation.

Si l'installation, en tant qu'objectif, n'est jamais remise en question dans les discours des responsables de syndicats d'exploitants ou d'organisations professionnelles agricoles, la volonté réelle d'installer au sein des départements est fréquemment discutée. De vifs désaccords apparaissent quand aux moyens à mettre en œuvre et à l'efficacité des différentes actions menées, tant au niveau du département qu'au sein de la CDOA, notamment concernant les modalités d'application du contrôle des structures.

Une des limites souvent signalée en matière de contrôle des structures, est la mauvaise information (ou l'absence d'information) des candidats à l'installation sur les terres libérées qui vont à l'agrandissement. Aussi le décret 99-964 du 25 novembre 1999 avait-il prévu une publicité obligatoire pour certaines autorisations d'exploiter, afin d'améliorer la connaissance des disponibilités en foncier et permettre aux jeunes intéressés de se faire connaître $^{3}$. Dans quatre des six départements, les exploitations qui font l'objet d'une

3. L'article R331-5 du Code rural créé par le décret 99-964 du 25 novembre 1999 prévoyait la publication, dans un journal local au moins, de la localisation et de la superficie des biens faisant l'objet d'une demande d'autorisation d'exploiter d'une superficie supérieure à la moitié de l'UR et n'ayant pas fait l'objet au préalable d'une inscription sur le répertoire à l'installation, ainsi que de l'identité des propriétaires ou de leurs mandataires. Cette obligation de publicité a été supprimée par le décret 2007-865 du 14 mai 2007 qui dispense de soumettre dans certaines conditions les autorisations d'exploiter à l'avis de la CDOA. demande d'autorisation d'exploiter sont mises en relation avec les candidats inscrits au Répertoire départemental à l'installation (RDI) : on cherche par ce biais à compenser l'offre trop limitée en exploitations du répertoire. Dans l'Aveyron, les données du RDI sont mêmes présentées systématiquement tous les six mois en CDOA, par l'Association départementale pour l'amélioration des structures des exploitations agricoles (ADASEA).

\section{Le rôle des syndicats dans les politiques d'installation}

\section{La représentation des différents syndicats dans les Commissions}

Les politiques d'installation et de contrôle des structures sont généralement traitées par les sous-commissions structures et économie des CDOA. Leur composition ne comporte que des représentants de l'agriculture et de l'administration et est représentative de la part des votes obtenue aux dernières élections des Chambres d'agriculture (la FDSEA et les JA totalisaient $62,5 \%$ des sièges de ces CDOA et avaient obtenus $60 \%$ des votes sur les 6 départements en 2001) (tableau 1). Cependant la présence en CDOA de représentants professionnels de la Chambre d'agriculture, de l'ADASEA ou d'autres Organisations professionnelles agricoles (OPA) confère à ces deux syndicats une large majorité des sièges, ce qui est fréquemment critiqué par les syndicats minoritaires, qui ont le sentiment d'être exclus du débat, de n'être pas écoutés en CDOA. En revanche ils ne remettent jamais en cause l'intérêt de leur participation aux commissions. Dans la Somme, l'Aveyron et le Morbihan, là où le contrôle des structures est appliqué le plus rigoureusement, les représentants des propriétaires semblent être en conflit avec les représentants de la profession agricole. À l'inverse dans les trois autres départements, les représentants des propriétaires ont le sentiment d'être entendus.

C'est dans la sous-commission «struc- 
RECHERCHES

Valérie BERNARDI, Jean-Pierre BOINON

Tableau 1. Résultats des élections Chambres d'agriculture et composition de la sous-commission « structures " de la $\operatorname{CDOA}^{4}$ (2001)

\begin{tabular}{l|c|c|c|c|c|c}
\hline & Somme & Aveyron & Morbihan & Eure & Finistère & $\begin{array}{c}\text { Haute- } \\
\text { Vienne }\end{array}$ \\
\hline FDSEA & $61,3 \%$ & $56 \%$ & $52,5 \%$ & $61,8 \%$ & $63,3 \%$ & $65,8 \%$ \\
\hline Confédération paysanne & $16,9 \%$ & $44 \%$ & $28,6 \%$ & $18 \%$ & $33,7 \%$ & $34,2 \%$ \\
\hline Coordination rurale & $21,9 \%$ & - & $15,2 \%$ & $20,2 \%$ & $3 \%$ & - \\
\hline Modef & - & - & $3,7 \%$ & - & - & - \\
\hline Nombre de représentants & 5 FDSEA & 8 membres des & 3 FDSEA & 4 FDSEA & 3 FDSEA & 2 FDSEA \\
par syndicat en & et JA & organisations & 2 JA & 2 JA & 2 JA & 2 JA \\
Commissions « structures » & 2 CR & Syndicales & 2 CP & 2 CP & 3 CP & 4 CP \\
& 1 CP & (JA, FDSEA, CP) & 1 CR & & & \\
\hline
\end{tabular}

Source : Ministère de l'Agriculture et enquêtes des auteurs auprès des DDAF

tures » qu'apparaissent le plus vivement les oppositions entre syndicats d'exploitants agricoles. Les désaccords sont réels mais non systématiques, et leur niveau peut aller du conflit ouvert concernant l'orientation à donner à la politique agricole départementale (par exemple dans le Finistère, qui est le seul des six départements à avoir vécu une alternance de majorité entre la FDSEA et la Confédération paysanne), au « consensus mou » signalé en Haute-Vienne. Dans tous les cas, on ne peut pas parler de blocages en CDOA : si les syndicats majoritaires et les représentants des différentes OPA trouvent un accord, en cas de vote ils forment une importante majorité. Les différences que nous avons observées tiennent donc plus au contexte des différents départements, qu'aux orientations politiques définies à un niveau national

\section{Le poids et le rôle des différents syndicats pour chaque département}

Les syndicats ont un rôle important à jouer dans le bon fonctionnement des CDOA, de part les débats qu'ils suscitent mais également en raison de leur implication locale

4. Par comparaison, les résultats aux élections de 2007 dans ces départements sont les suivants : Somme, Aveyron, Morbihan, Eure, Finistère, HauteVienne, FDSEA : 72,5 \% 55,8 \% 54,2 \% 59,7\% $60,4 \% 77 \%$; Confédération paysanne : $6,7 \%$ $22,8 \% 25 \% 12,4 \% 22,5 \% 23 \%$; Coordination rurale : $20,7 \% 21,3 \% 20,7 \% 27,8 \% 17 \%$ (source : ministère de l'Agriculture). indispensable à la diffusion de l'information. Ainsi, une meilleure communication pour diffuser l'information concernant les demandes d'autorisation d'exploiter, l'élargissement des commissions à des acteurs de la société civile, un plus grand accompagnement des projets diversifiés ou innovants sont quelques-unes des suggestions des syndicats pour une plus grande efficacité des CDOA.

Nous présenterons d'abord l'analyse syndicale des principaux freins à l'installation, par département. Puis, nous verrons de quelle manière les syndicats s'investissent dans les politiques d'installation départementale.

\section{Les principaux freins à l'installation}

Parmi les principaux freins à l'installation cités par les syndicats, le problème de la pression foncière est largement évoqué par la FDSEA que ce soit en Aveyron (en raison de la proximité avec Montpellier), dans le Finistère (en raison des contraintes environnementales), en Haute-Vienne (pression sur l'habitat par les résidences secondaires) ou dans le Morbihan. Dans l'Eure, le problème du prix des reprises qui ne permet l'installation qu'aux fils d'agriculteurs inquiète beaucoup, notamment la Confédération paysanne. La FDSEA cite également pour la Somme le problème du coût des reprises qui échappe à la CDOA et de la difficulté à rendre efficace le contrôle des structures. Plus que l'arbitrage de la CDOA, 
c'est ici le consensus professionnel sur la gestion du foncier, par le travail des commissions cantonales réunissant la FDSEA et les JA, qui participe au taux de renouvellement observé. La barrière de contrôle des structures permet alors de limiter les risques de surenchère, qui bloquerait l'installation ou hypothéquerait la viabilité future de l'exploitation. Enfin, selon les JA, en HauteVienne, les difficultés économiques de la filière viande bovine découragent les nouvelles générations d'agriculteurs à s'installer, lesquelles préfèrent prendre le statut de salarié agricole, et éviter ainsi les risques de faillite et les tracasseries administratives.

\section{La publicité concernant les demandes d'autorisation d'exploiter}

Le pourcentage de dossiers en concurrence pour l'obtention de l'autorisation d'exploiter sur des mêmes parcelles est extrêmement variable d'un département à l'autre. Les deux départements bretons se caractérisent par des taux élevés de dossiers en concurrence (supérieurs à $30 \%$ ), ce qui permet à la CDOA de mettre en œuvre son rôle d'arbitrage entre les différentes demandes et peut ainsi être l'occasion de définir une stratégie concernant les orientations possibles du foncier. La publicité réalisée dans la presse agricole locale, obligatoire lors de nos enquêtes pour les surfaces supérieures à $1 / 2$ SMI non inscrites au RDI, peut susciter des demandes de foncier et augmenter ainsi le pouvoir d'arbitrage de la CDOA. Le décalage entre le type d'offre d'exploitation au RDI et le type de demande est souvent regretté : la FDSEA du Finistère pense que ce problème pourrait être amélioré si les démarches étaient intensifiées pour rechercher des exploitations correspondant à la demande, notamment des petites structures pour des projets diversifiés. Dans ce département, les projets qui ne rentrent pas dans le cadre des systèmes de production agricole dominants (projets diversifiés ou innovants, avec transformation et commer- cialisation) trouvent difficilement un appui technique. Généralement, les représentants de la Confédération paysanne se prononcent pour un développement de la publicité des demandes d'autorisation d'exploiter. Cette publicité est réalisée par la DDAF dans l'ensemble des départements sauf dans la Somme, où les avis de demande d'autorisation d'exploiter sont simplement affichés en mairie. Ici, l'absence de publicité dans la presse est assumée et argumentée par les responsables professionnels majoritaires, selon lesquels la concurrence entraînerait une hausse des prix qui n'est pas souhaitable. Pour remédier à cette situation, la Confédération paysanne de la Somme propose que l'inscription au RDI soit rendue obligatoire durant une période donnée. Au-delà de la disponibilité de l'information, d'autres facteurs limitent donc la tendance des candidats à se porter concurrents, comme des montants de reprise dissuasifs, ou une organisation locale qui ferait que seule la personne la mieux placée formule sa demande. L'absence de publicité, ou sa faible amplitude, limiterait donc l'accès à l'information principalement pour des candidats venant de l'extérieur.

\section{L'importance des relais locaux dans la mise en auvre du contrôle des structures} La DDAF transmet les dossiers aux membres des CDOA, entre huit et quinze jours avant que la commission ait lieu, intervalle de temps dont disposent les membres de la CDOA pour instruire les dossiers. Les représentants des syndicats transmettent l'information relative aux dossiers à leurs délégués locaux ou cantonaux. Cependant, les relais locaux prennent plus ou moins d'importance selon les départements et les syndicats. En effet, les syndicats minoritaires sont rarement suffisamment développés pour posséder un réseau d'adhérents qui couvre tout le territoire, et qui ait la possibilité de faire remonter des avis. La Coordination rurale ne possède pas de réseau 


\section{RECHERCHES}

de ce type dans les départements étudiés, et la Confédération paysanne ne transmet des avis que dans le Morbihan, l'Eure et le Finistère. Le cas de la Haute-Vienne est à ce titre particulier puisque des comités locaux à l'installation, certes peu actifs, regroupent les différents syndicats. La Confédération paysanne souhaiterait qu'ils soient plus actifs afin de mettre réellement les dossiers en concurrence. Dans tous les départements, des relais sont mis en place conjointement par la FDSEA et les JA. Les trois départements qui présentent les taux de renouvellement les plus bas sont aussi ceux dans lesquels ces relais apparaissent les moins structurés et peu d'avis parviennent en CDOA. Dans les trois autres départements, ces avis locaux sont presque systématiques (dans l'Aveyron les maires sont également consultés), et visent à prendre en compte la situation réelle des exploitants concernés. Des groupes de travail composés de l'administration, des syndicats majoritaires et de représentants des autres organismes agricoles (ADASEA, SAFER, Chambre d'agriculture...) se réunissent avant chaque CDOA pour discuter des avis cantonaux et prendre une position commune avant la CDOA. Ces réunions permettent d'extraire des dossiers les éléments de comparaison, mais aussi de détecter les exploitations qui pourraient permettre une installation. Ainsi, dans ces départements, la majeure partie des débats a déjà eu lieu avant la réunion de la $\mathrm{CDOA}$, en groupe restreint qui exclut notamment les syndicats minoritaires. Si pour la FDSEA de l'Aveyron, l'efficacité du contrôle des structures n'est possible que grâce au travail réalisé en amont, la Confédération Paysanne de ce département critique vivement le pouvoir des syndicats dominants. Elle juge notamment que le traitement des cas de concurrence relève du clientélisme local. Ce point de vue est partagé par la Propriété agricole, qui est opposée au contrôle dans son ensemble.

\section{Conclusion}

Parce qu'elles ont la possibilité d'orienter l'évolution des structures des exploitations agricoles d'un département, les CDOA représentent un enjeu fort pour le syndicalisme agricole et l'attribution des sièges représentant les différentes organisations professionnelles agricoles dépend du résultat des élections aux Chambres d'agriculture.

Mais les CDOA sont des instances difficiles à gérer du fait du nombre important et de l'hétérogénéité de ses membres, mêmes si elles représentent de véritables structures de débats et de concertation entre les représentants élus des agriculteurs et les partenaires de l'agriculture. Une analyse approfondie du fonctionnement dans six départements français, choisis par rapport à leur système de production dominant et de l'implication des syndicats départementaux dans la mise en œuvre d'une politique plus ou moins volontariste en matière d'orientation des structures des exploitations agricoles, nous a permis de mettre en évidence l'intérêt de cette institution pour orienter l'agriculture départementale, mais aussi un certain nombre de limites.

$$
* \quad *
$$

Ainsi, on peut noter le rôle déterminant du syndicalisme majoritaire (FNSEA et JA) qui, bénéficiant de relais syndicaux sur tout le territoire, peut se donner les moyens d'instruire les dossiers et, selon les cas, choisit ou non de les mobiliser. Ces deux syndicats bénéficient également des infrastructures des autres organisations professionnelles agricoles qu'ils contrôlent. En regard, l'action de la Confédération paysanne, malgré l'investissement personnel de ses militants, peut apparaître d'une plus faible efficacité, ce qui entraîne parfois un certain sentiment de lassitude. Dans aucun des départements étudiés, la Coordination rurale ne s'est donnée les moyens de pouvoir intervenir dans la politique d'installation. 
Tout comme les représentants de la Propriété agricole, elle s'oppose généralement au contrôle des structures, qu'elle considère comme un outil de clientélisme pour les syndicats majoritaires. Les principales différences de comportement sont observées à l'intérieur même du syndicalisme majoritaire, dont les représentants dans certains départements appliquent une réelle politique favorisant l'installation de jeunes agriculteurs, tandis que dans d'autres, ils laissent jouer plus librement les règles du marché dans l'attribution de moyens de production aux jeunes agriculteurs. Le rôle des CDOA a été vivement discuté dans les débats régionaux préparatoires à la loi d'orientation agricole du 5 janvier 2006 et le Comité national d'orientation (CNO) de l'Agriculture, dans sa synthèse des débats proposait au législateur de "limiter voire même de supprimer le rôle des CDOA en matière de traitement individuel des dossiers », même s'il notait que les positions étaient partagées selon les régions et que certaines demandaient « un renforcement du pouvoir décisionnel de la CDOA [...], dans le but d'une meilleure adaptation locale et de promouvoir l'installation » (CNO, 2004).

\section{RÉFÉRENCES BIBLIOGRAPHIQUES}

Barthélemy D. (2001). L'installation des jeunes et le patrimoine professionnel en agriculture. Paris, Actes et communications, $\mathrm{n}^{\circ} 18$, INRA.

Barthélemy D., David J. (Ed.) (1999). L'agriculture européenne et les droits à produire. Paris, INRA, $434 \mathrm{p}$.

Berriet-Solliec M., Boinon J.-P. (2000). Analyse des instruments d'orientation de l'agriculture départementale. Paris, Économie Rurale, n² 260, p. 148-153.

Cahiers du CNASEA (2002). L'installation en agriculture : analyse et perspective. Limoges, consulté le 20 mars 20007 (http://www.cnasea.fr/accueil/cadre_ publications.html), $25 \mathrm{p}$.

CNO (2004). Agriculture, Territoires et Société. Synthèses des débats régionaux. Consulté le 16 février 2005 (http:// www.agriculture.gouv.fr/spip/IMG/pdf/ com_cnoavis201204.pdf), 2 vols. 24 et $48 \mathrm{p}$.

Coulomb P. (1990). La cogestion : une nouvelle tentative corporatiste ? In Coulomb P., Delorme H., Hervieu B.,
Jollivet M., Lacombe P., « Les agriculteurs et la politique Paris FNSP », p. 147-158.

Debatisse M. (1963). La révolution silencieuse : le combat des paysans. Paris, Calmann-Lévy, $275 \mathrm{p}$.

Hottlet L. et CNJA (2001). L'installation des jeunes agriculteurs. $2^{\mathrm{e}}$ édition, Éditions Agridécisions, $140 \mathrm{p}$.

Lefevre F. (2004). Vers un boum des installations grâce au départ à la retraite de la génération du baby-boum? Limoges, CNASEA, mai, 5 p.

Machline E. (2005). Mise en ouvre des politiques d'installation par les CDOA: analyse comparative dans 6 départements. Limoges, CNASEA, 2 Tomes, 87 et 97 p.

Rémy J. (1997). Les sans-dot de l'agriculture : faut-il aider les installations sans aide ? Économie Rurale, $\mathrm{n}^{\circ} 238$, p. 33-37.

Vercherand J. (2009). Analyse économique du syndicalisme agricole (Une approche typologique et comparative à partir des défaillances de marché). Économie Rurale (à paraître). 